\title{
An Adaptive Merging Method between Point Clouds in Reverse Engineering
}

\author{
Peng-Xin LIU1,a, Meng WU1', Yun-Qin ZHU², Hai-Li JIA', Jia-Yin ZHAO' \\ 1 Tianjin Key Laboratory of High Speed Cutting and Precision Machining, Tianjin University of \\ Technology and Education, 300222 Tianjin, China. \\ 2Jimei Industry College, no.22 xingqian road. xiamen city .fujian province. China.
}

Keywords: Reverse engineering, Adaptive merging, Point clouds.

\begin{abstract}
This paper introduces an adaptive merging method between point clouds from optical scanning system. The point clouds will be wrapped in the form of triangulated mesh. One is used as feature data, and the other point cloud as target data. Then the special mesh region that needs to be updated is automatic recognized through the detection of the overlapping area between the feature data and target data, and the half edge data structure and angle criterion is used. The target triangular mesh is eventually updated to the final output by replacing the recognized region with the newly added feature data points. After that, the merging data import to CAD software environment to reconstruct. Experimental results demonstrate the efficiency of the proposed method, which is suitable to rapid customization and deformation of triangular mesh model for the product innovative design with the shorten time.
\end{abstract}

\section{Introduction}

Reverse engineering can be defined as the technology of generating a digital model from an existing part or prototype by computer aided design and digital measurement. While reverse engineering has been used for many years in industry, particularly in the automotive and aerospace sectors, it has shown a marked increase in implementation [1]. With these, the product design and manufacturing cycle has shown time reduction [2].This is primarily due to the development of rapid part digitization technologies and the availability of inexpensive computers. In fact, reverse engineering is becoming widely used in the fields of medicine, rapid prototype manufacturing, film, innovative design and so on.

The most widespread digitization system in reverse engineering is noncontact system based on optical technologies, which is much more efficient in terms of speed and reducing the human labour. In the last decade, a large amount of work has been performed to develop methods for the mathematical modelling of optical scanning 3D points, in order to facilitate handling of them on existing CAD/CAM systems [3]. This paper mainly focuses on date merging for free-form surfaces which is the key technology in the combined RE modelling method. The whole process is shown in the figure 1.

The starting point is the acquisition of a number of clouds of points using optical scanning probe from the different part. One point cloud is used as feature data, and the other point cloud as target data. These two point clouds are exported in the form of a triangulated mesh, respectively. First, identify the boundary points of feature data, by means of half edge data structure to grid model identifies boundary triangle, and then extract boundary ring based on the judgment theory of point in-or-out of polygon, realizes adaptive mesh segmentation based on the boundary ring. The recognition of regional integration is realized under the angle criterion. This paper proposed an adaptive merging method between different point clouds, which is very meaningful for the product innovative design. 


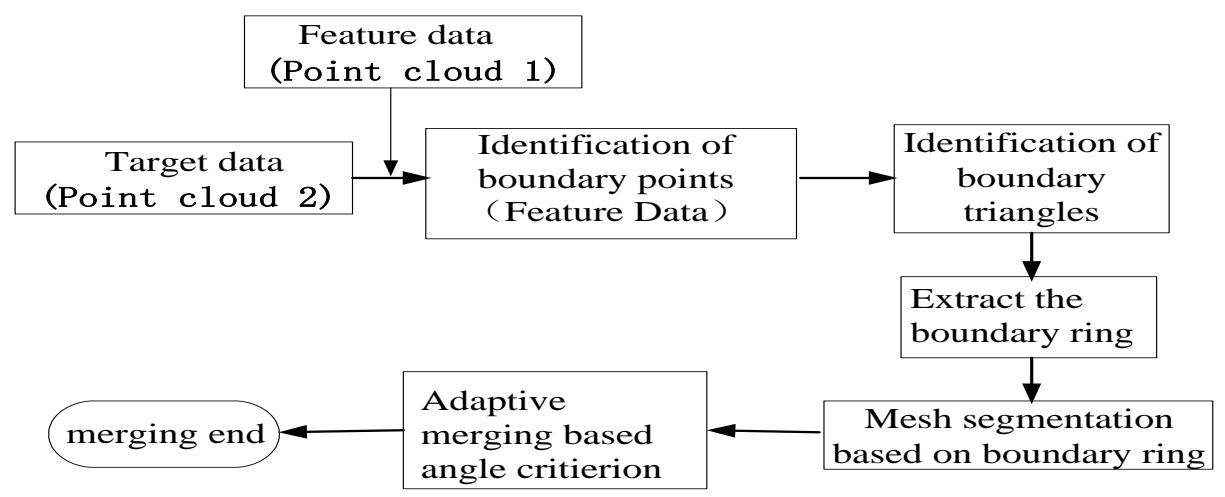

Figure 1. Overall procedure for data merging

\section{Identification of Boundary Triangles}

In order to extract the boundary ring, first need to identify boundary points of feature data, which is the measuring point can express real boundary characteristics.

\section{Identification of Boundary Points}

The halfedge data structure[4] is used, as shown in Figure 2.

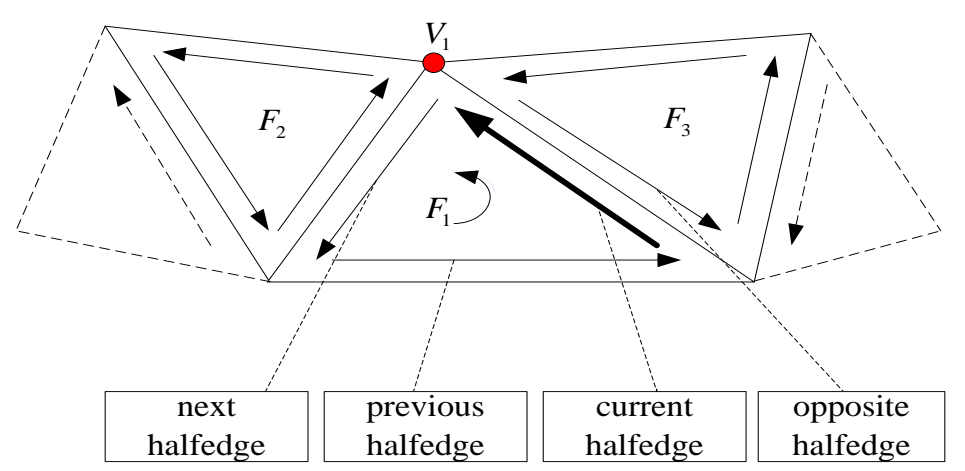

Figure 2. The data structure of triangle mesh

A halfedge data structure is an edge-centered data structure capable of maintaining incidence information of vertices, edges and faces. Each edge is decomposed into two halfedges with opposite orientations. Each triangle is composed of three halfedges, and the topological structure of import triangle mesh is built through the adjacent half edge. It will be easy to find the connected half edge and triangles of the known point. The data structure is as follows.

Struct HalfEdge \{ int halfEdgeID;

int triID;

Point *pEnd; int preHalfEdgeID;

int nextHalfEdgeID;

int vsHalfEdgeID;

The halfEdgeID and triID represents the index of a half edge and the triangle that the half edge belonged, respectively. The preHalfEdgeID represents the index of the previous halfedge. The nextHalfEdgeID represents the index of the next halfedge. The pEnd is the pointer points the endpoint of the halfedge.

The opposite halfedge index expressed as vsHalfEdgeID, and the default value is -1 .With the building of halfedge data structure, the vsHalfEdgeID value of each edge will be reset unless the boundaries. The vertex is considered the boundary point, as long as a vertex connected to the edge that the vsHalfEdgeID value is -1 . 


\section{Indentifcation of Boundary Triangles}

Boundary points will be project to the target triangle mesh. Based on sorting of the projection point, formation sequence $\mathrm{P}_{\mathrm{i}}, \mathrm{i}=0,1,2 \ldots \mathrm{n}$, the number of projection points is $\mathrm{n}+1$, connected projection points according to the sequence, such as the formation of polygon $\mathrm{P}=\mathrm{P}_{0}, \mathrm{P}_{1}, \mathrm{P}_{2}, \ldots, \mathrm{Pn}$ is shown in Figure 3.

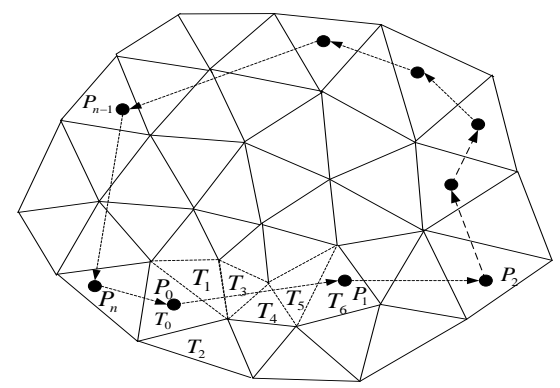

Figure 3. The data structure of triangle mesh

If the projection area is a plane, polygon $\mathrm{P}$ is a planar polygon. For example, projection point $\mathrm{P}_{0}$ and $\mathrm{P}_{1}, \mathrm{P}_{0}$ corresponds to the boundary triangle $\mathrm{T}_{0}, \mathrm{P}_{1}$ corresponding to the boundary triangle $\mathrm{T}_{6}$, edge $\mathrm{P}_{0} \mathrm{P}_{1}$ through the triangle (dashed line) $\mathrm{T}_{1}, \mathrm{~T}_{3}, \mathrm{~T}_{4}, \mathrm{~T}_{5}$ is the boundary triangle which need to be marked. Therefore, a criteria is given: if two points on either side of the triangle polygon $\mathrm{P}$ respectively in the internal and external, then the triangle connected the edge on both sides is boundary triangle.

If the projection area is not a plane, polygon $\mathrm{P}$ is not a planar polygon. There is no guarantee that the edges of the polygon $\mathrm{P}$ have an intersection point with the target triangle mesh. However, the direction of the polygon $\mathrm{P}$ will not change, so project the vertices of polygon $\mathrm{P}$ to the target triangle mesh, and the projection points instead of the vertices, as show in figure $4, v_{2} v_{3}$ is the growth edge, $T_{\text {neigh }}$ is the boundary triangle of new mark. Project point $P_{\mathrm{i}-1}, P_{\mathrm{i}}$ to the triangle $T_{\text {neigh, }}$ and get the protection points $Q_{\mathrm{i}-1}, Q_{\mathrm{i}}$, replace the points $P_{\mathrm{i}-1}, P_{\mathrm{i}}$, then find the new growth edge. It is worth noting that $T_{\text {neigh, }}$ is a variable, and represent the boundary triangle of new mark between $T_{\text {pre }}$ and

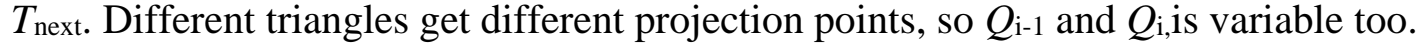

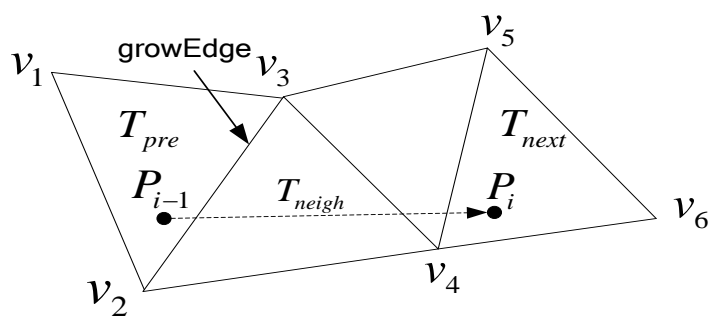

Figure 4. The position relation of adjacent projected points

The experiment also found that the projection points $Q_{\mathrm{i}-1}$ and $Q_{\mathrm{i} \text {, on the }}$ triangle $T_{\text {neigh }}$ of points $P_{\mathrm{i}-}$ 1 or $P_{\mathrm{i}}$ overlap with the vertex of $T_{\text {neigh }}$ in rare cases, shown in figure 5 . So, it can not find the intersection point between the line $Q_{\mathrm{i}-1} Q_{\mathrm{i}}$, and the edges of the triangle $T_{\text {neigh }}$ unless the vertices, and the mass center of $T_{\text {neigh }}$ is used to replace the projection point. 


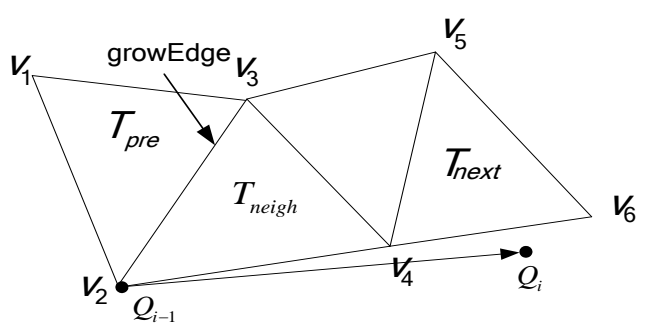

Figure 5. Coincidence of a projected point and a triangle vertex

\section{Extraction Boundary Ring}

When the boundary triangles are identified, closed boundary ring can be constructed through the extraction operation. In order to divide the model, first choose a triangle which does not belong to any boundary ring as seed triangle, then divided the triangle model by area growth method [5]. There are two categories of triangle in this paper, one is the marked triangle on the boundary ring, and the other is the non-boundary ring triangle. The target triangular mesh will be divided into different areas through the boundary rings.

In the process of area growth, the adjacent triangles with the same mark are integrate into the patch with the seed triangle continuously, until the adjacent triangles are all becoming the boundary ring, then to the other side of the boundary ring, the adjacent triangle which is non boundary ring becoming the new seed triangle, repeat the area growth process. The initial triangular mesh model is divided into $\mathrm{k}$ patches at last. $\mathrm{k}$ is the index of patch, $\mathrm{k}=0,1,2 \ldots \mathrm{m}+1, \mathrm{~m}$ is the number of boundary ring. In the $\mathrm{k}$ patch contains the point which need to be replaced by the feature data. This process need to be judged by certain criteria, the angle criterion which will be discussed in the following sections of this paper.

\section{Adaptive Data Merging Based Angle Criterion}

The boundary ring is the overlap area between the feature data and the target data; these two areas will coincide at the boundary in measuring process at least. The angle between the boundary point of feature data and the surface (waiting fused area) within the boundary ring is less then the angle between the boundary point of feature data and the other surface.

Figure 6 is an example of the angle, point A, B as the projection point in the boundary ring, $\theta 1$ is a angle between the boundary point of feature data and the surface within the boundary ring, which is less than the $\theta 2$ ( the angle between the boundary point of feature data and the outside surface of boundary ring).

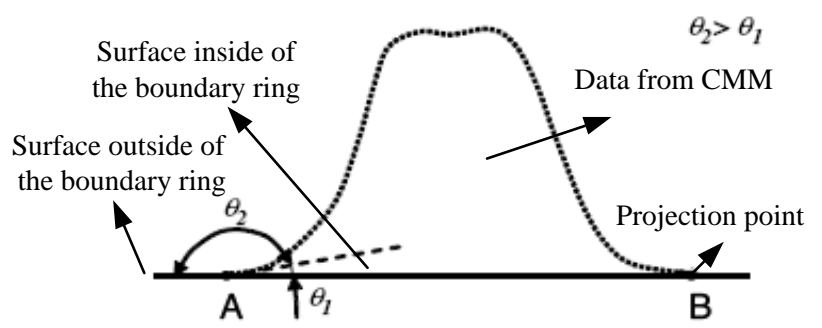

Figure 6. An example of the angle condition

In order to description the algorithm facilitated, gives the following symbols and definitions:

$E_{i}\left(P_{i}, P_{i+1}\right)$ : The line between adjacent projection points $P_{i}, P_{i+1}$ within the boundary ring, also called the boundary edge.

$T_{i}^{k}$ : The triangle which formed with the boundary edge $E_{i}$ and the nearest point to the midpoint of $E_{i}$, where $k$ is the patch index, $\mathrm{i}$ is boundary edge index, as the triangle $T_{i}^{c}$ and $T_{i}^{l}$ in Figure 7. 
$F_{i}{ }^{m}$ : The triangle is formed by the boundary edge $E_{i}$ and CMM measurement data which is nearest to the midpoint of $E_{i}$ and not a boundary edge point, where $\mathrm{m}$ is the number of CMM data sets, $\mathrm{i}$ is the boundary edge index.

$T_{i}^{k}$ and $F_{i}{ }^{m}$ are called shared boundary edge triangles

$\theta_{i}{ }^{k}$ : It's the angle between the shared boundary edge triangles $T_{i}^{k}$ and $F_{i}{ }^{m}$, also called boundary angle.

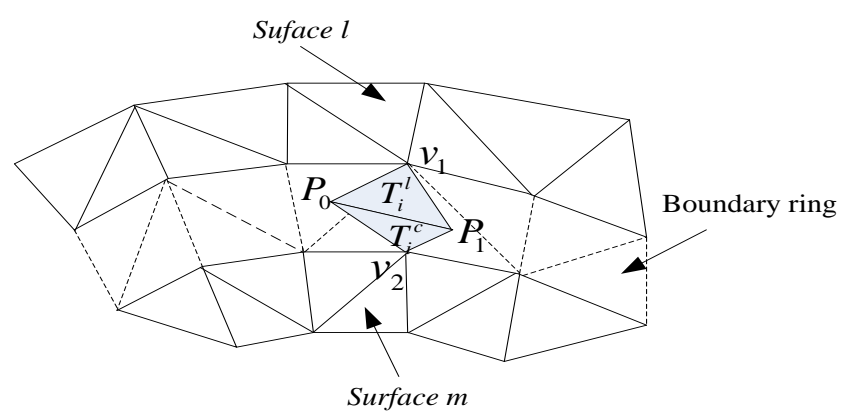

Figure 7. Shared triangle of boundary edge

$\theta_{i}{ }^{k}$ is calculated by the normal vector of $T_{i}{ }^{k}$ and $F_{i}{ }^{m}$, it's should be consistently in the process. for example, in Figure 8, the formula of normal vector of triangle $T_{i}^{c}, T_{i}^{l}, F_{i}{ }^{m}$ is as followed:

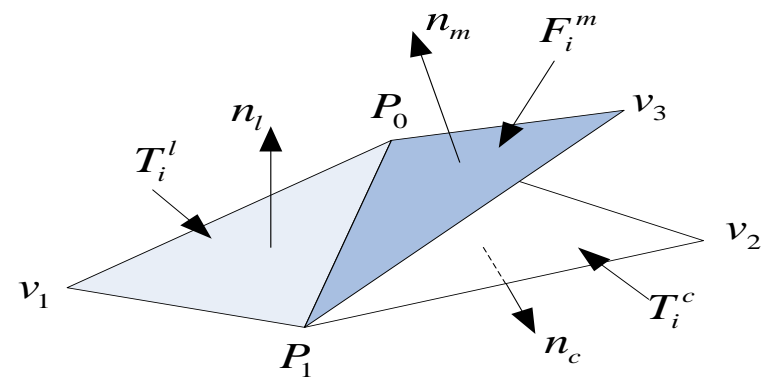

Figure 8. Relationship between the boundary angle

$$
\begin{aligned}
& n_{c}=\frac{P_{0} v_{2} \times P_{0} P_{1}}{\left|P_{0} v_{2} \times P_{0} P_{1}\right|} \\
& n_{l}=\frac{P_{0} v_{1} \times P_{0} P_{1}}{\left|P_{0} v_{1} \times P_{0} P_{1}\right|} \\
& n_{m}=\frac{P_{0} P_{1} \times P_{0} v_{3}}{\left|P_{0} P_{1} \times P_{0} v_{3}\right|}
\end{aligned}
$$

The formula for $\theta_{i}{ }^{k}$ is :

$$
\theta_{i}^{k}=\angle F_{i}^{m} T_{i}^{k}=\cos ^{-1}\left(\frac{n_{F_{i}^{m}} \bullet\left(-n_{T_{i}^{k}}\right)}{\left|n_{F_{i}^{m}}\right|\left|\left(-n_{T_{i}^{k}}\right)\right|}\right)
$$

$\theta_{i}{ }^{k} \leq 0 \leq \pi, \mathrm{i}=0, \ldots, \mathrm{n}, \mathrm{n}$ is the number of boundary edge. According to the boundary angle $\theta_{i}{ }^{k}$, the following decision angle criterion of regional integration is given:

Suppose $\theta_{\text {mean }}$ is the average of $\theta_{i}{ }^{k}$, then: 


$$
\theta_{\text {mean }}^{k}=\sum_{i=0}^{n} \theta_{i}^{k} / n
$$

$k$ is patches index, $n$ is the number of boundary edges, has known the patches which the vertex is nearest to the boundary edge are $l$ and $c$, and $\theta_{\text {mean }}^{l}<\theta^{c}$ mean, then $l$ is the patch merging region.

According to the angle criterion, marked the merging region. the target data at merging region will be deleted, replaced by feature data. Finally, the complete CAD model will be reconstruct based on these merging data under the optimization of reconstruction strategy

\section{Results and Discussion}

Using the algorithm of this paper, one experiments have done to verify the theory. Figure 9 is a model of data fusion instance.

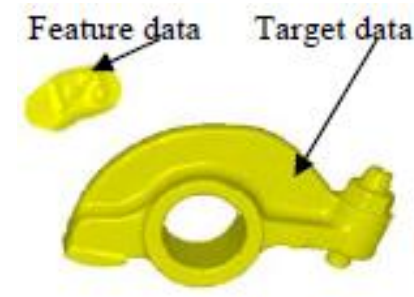

a) The initial mesh model and data added

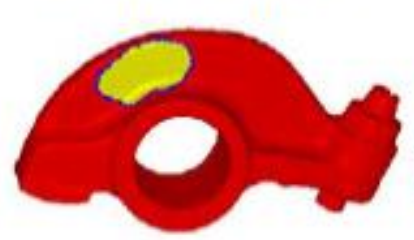

c) Mesh segmentation based on boundary ring

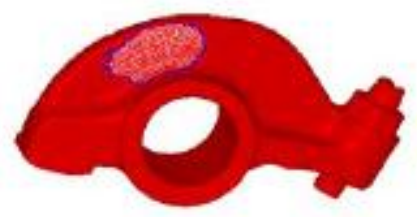

e) The results of data fusion

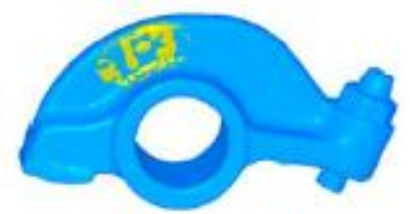

b) After registration

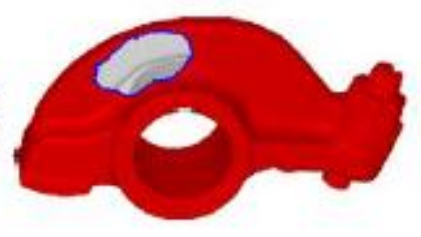

d) Delete the fusion region

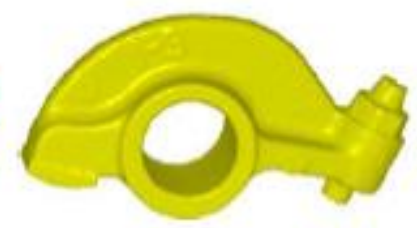

f) The updated mesh model

Figure 9. An example of a rockerarm data merging

Figure 9 a) as the initial triangular mesh model of the rocker arm and point data need to be added at some surface, the results that registrated is shown in Figure $9 \mathrm{~b}$ ). Mesh segmentated based on the boundary rings is shown in Figure 9 c), Figure 9 d) is the results after deleting the overlap region, the results of data fusion is shown in Figure 9 e). Figure $9 \mathrm{f}$ ) is the updated triangle mesh model based on the feature data. From this example, the method in this paper can realize the rapid customization and deformation design of initial mesh model with less digitization time.

\section{Conclusions}

An adaptive merging method between point clouds is proposed in this paper. The overlapping regions between feature data and target data from optical scanning are identified. The target data is updated adaptively according to the feature data. The proposed method is suitable to achieve rapid customization of triangular mesh model and deformation. Time for the digital design is shortening 
too, because there is no need to rescan all the part in this method. It's meaningful for the product innovative design.

\section{Acknowledgements}

This work was financially supported by the Natural Science Foundation of Tianjin (15JCQNJC05200) and Tianjin University \& Education Foundation (KJ1423) and Tianjin University \& Education Foundation (KJ1309)

\section{References}

1. Ye, X. Z., Liu, H.Z., and Chen, L., et al. Computer Aided Design (2007)

2. Kuo, C. -C., Yau, H. -T.. Journal of Computer and Information Science and Engineering (2003).

3. Ke, Y. L., Fan, S. Q., Zhu, W. D., et al. Computer Aided Design (2003).

4. http://www.openmesh.org/DailyBuilds/Doc/a00016.html

5. H.W.Lin, C.L.Tai, G.J.Wang. Computer-Aided Design(2004) 